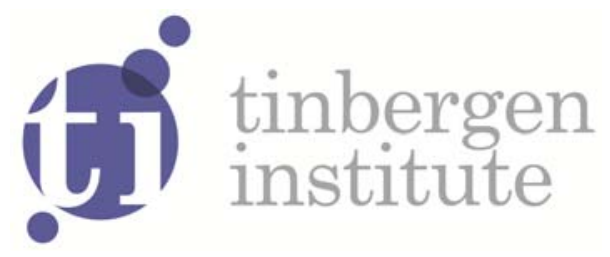

\title{
Bayesian Forecasting of US Growth using Basic Time Varying Parameter Models and Expectations Data
}

\author{
Nalan Basturk ${ }^{1}$ \\ Pinar Ceyhan²,3 \\ Herman K. van Dijk $k^{2,3,4}$
}

1 Maastricht University, the Netherlands;

2 Erasmus School of Economics, Erasmus University Rotterdam, the Netherlands;

3 Tinbergen Institute, the Netherlands

4 Faculty of Economics and Business Administration, VU University Amsterdam, the Netherlands. 
Tinbergen Institute is the graduate school and research institute in economics of Erasmus University Rotterdam, the University of Amsterdam and VU University Amsterdam.

More TI discussion papers can be downloaded at http://www.tinbergen.nl

Tinbergen Institute has two locations:

Tinbergen Institute Amsterdam

Gustav Mahlerplein 117

1082 MS Amsterdam

The Netherlands

Tel.: +31(0)205251600

Tinbergen Institute Rotterdam

Burg. Oudlaan 50

3062 PA Rotterdam

The Netherlands

Tel.: +31(0)10 4088900

Fax: $+31(0) 104089031$

Duisenberg school of finance is a collaboration of the Dutch financial sector and universities, with the ambition to support innovative research and offer top quality academic education in core areas of finance.

DSF research papers can be downloaded at: http://www.dsf.nl/

Duisenberg school of finance

Gustav Mahlerplein 117

1082 MS Amsterdam

The Netherlands

Tel.: +31(0)20 5258579 


\title{
Bayesian Forecasting of US Growth using Basic Time Varying Parameter Models and Expectations Data
}

\author{
Nalan Basturk ${ }^{1}$, Pinar Ceyhan ${ }^{2,3}$, and Herman K. van Dijk $k^{2,3,4}$ \\ ${ }^{1}$ Department of Quantitative Economics, Maastricht University \\ ${ }^{2}$ Econometric Institute, Erasmus University Rotterdam \\ ${ }^{3}$ Tinbergen Institute \\ ${ }^{4}$ Department of Econometrics, VU University Amsterdam
}

\begin{abstract}
Time varying patterns in US growth are analyzed using various univariate model structures, starting from a naive model structure where all features change every period to a model where the slow variation in the conditional mean and changes in the conditional variance are specified together with their interaction, including survey data on expected growth in order to strengthen the information in the model. Use is made of a simulation based Bayesian inferential method to determine the forecasting performance of the various model specifications. The extension of a basic growth model with a constant mean to models including time variation in the mean and variance requires careful investigation of possible identification issues of the parameters and existence conditions of the posterior under a diffuse prior. The use of diffuse priors leads to a focus on the likelihood function and it enables a researcher and policy adviser to evaluate the scientific information contained in model and data. Empirical results indicate that incorporating time variation in mean growth rates as well as in volatility are important in order to improve for the
\end{abstract}


predictive performances of growth models. Furthermore, using data information on growth expectations is important for forecasting growth in specific periods, such as the the recession periods around 2000s and around 2008 .

JEL codes: C11, C22, E17

Keywords: Growth, Time varying parameters, expectations data.

\section{Introduction}

Quarterly economic growth in the USA, measured as the quarterly change in log real Gross Domestic Product, has shown typical data features in the time period 1947QI until 2013QIV with, as most important ones, a time varying mean and variance. It is important to model these stylized data features which in turn may serve as inputs for forecasting growth gaps and/or as indications of the development of economic welfare. These data features have been analyzed in many papers. A complete literature analysis is beyond the scope of this paper, but a summary is given in the next section.

In this paper these time varying patterns in US growth are analyzed using various univariate model structures for time variation, starting from naive model structures where all features change every period to a model where the slow variation in the conditional mean and changes in the conditional variance are specified together with their interaction. Use is made of a simulation based Bayesian inferential method to determine the forecasting performance of the various model specifications. The extension of a basic growth model with a constant mean to models including time variation in the mean and variance requires careful investigation of possible identification issues of the parameters and existence conditions of the posterior under a diffuse prior. The use of diffuse priors leads to a focus on the likelihood function and it enables a researcher and policy adviser to evaluate the scientific information contained in model and data. As Hildreth (1963) argued: 'Reporting the shape of the likelihood and its properties is an important task for a Bayesian econometrician.' For this reason, in section 3, these topics are analyzed and a 
connection is made with the well known Hierarchical Linear Mixed Model (HLMM), see Hobert and Casella (1996). Results are illustrated using simulated data.

Macroeconomic data are usually not so informative on detailed data characteristics and an additional source of information is the use of expectations data on growth, for instance, from the survey of professional forecasters; see Milani (2011). We make use of these data in section 5.5.

The use and analysis of flexible model structures about mean and variance of US economic growth and the use of expectations data allow one to compare empirical results obtained from alternative models. This gives information on their relative strengths and weaknesses with respect to posterior accuracy and predictive performance. As stated above such models may also serve as input for other purposes like estimating a growth gap.

The contents of this paper are organized as follows. Some stylized facts on the data are presented in section 2 together with a brief survey of the literature. In section 3 properties and shapes of the likelihood functions for some naive models for growth with time varying parameters are analyzed together with existence conditions of posteriors under diffuse priors. The proposed model extensions, and the properties of the posterior parameter distributions in each model are presented in section 4. Empirical results on prediction are presented in section 5 while section 6 presents some final remarks including suggestions for further research.

\section{Stylized facts about US real GDP growth}

As stated in the introduction, modeling quarterly change in the log of the US real GDP series, which has undergone periodic changes in the level during the post World War II period, is important for measuring and forecasting actual and potential US growth. Changes in the pattern of US real GDP growth have been modeled by various authors in the literature. We present a selective survey of papers that relate to the topic of this 
paper and refer further to the references cited in these papers. McConnell and PerezQuiros (2000) suggest that the volatility of US real GDP decreased in early 1980s and that there is a change in the mean in US real GDP growth that occurred in the first quarter of 1984. These authors model US GDP growth as a Markov switching model with two features: (i) the mean and variance follow independent switching processes, (ii) a two-state process for the mean changes according to the state of the variance. Blanchard and Simon (2001) report on the decline of US output volatility, suggesting a decline in the standard deviation of output fluctuations over time, from about $1.5 \%$ a quarter in the early 1950s to less than $0.5 \%$ in the late 1990s. Blanchard and Simon (2001) shows that this decline is not continuous since volatility increases from the late 1960s to the mid-1980s, and this is followed by a sharp decline in late 1980s. Their paper mentions two possible ways of modeling the output volatility: (i) a declining trend with interruptions in 1970s and early 1980s, and (ii) a downward shift in mid-1980s. Clarida et al. (2000) consider two sub-periods for different output volatility: (i) 1960Q1-1979Q2 (pre-Volcker) and (ii) 1979Q3-1996Q4 (Volcker and Greenspan). Kim et al. (2004) suggest that the decline in the volatility of the US real GDP growth beginning in the early 1980s is concentrated in the cyclical component rather than in the trend component. Penelope and Summers (2009) find a decline in the US GDP volatility beginning in late 1984, using a model in which the mean and variance of GDP growth are influenced by latent state variables following independent Markov chain processes. Kim and Nelson (1999) use a Bayesian approach to identify a change in the mean at an unknown change point in a Markovswitching model of the business cycle. Their paper suggests a decline in the variance of shocks and a change in the mean in 1984Q1. It also finds a narrowing gap between growth rates during recessions and booms. Several other authors studied US growth data but as indicated earlier a detailed analysis is beyond the scope of this paper. The lesson from this literature analysis is to seriously consider and model time varying parameter models in order to model the observed low and high frequencies in the data. 
Before the modeling step, it is useful to perform an exploratory data analysis on the data series that are extended over a longer period than the earlier studies listed above. An important stylized fact observed from Figure 1 about US real GDP growth is that the mean of the series is changing during the post WWII period, albeit not continuously like a random walk where at each period of time a shift in the series is observed. Instead, we observe occasional and discrete shifts in the mean of the series around data points like 1965 and 1984. The mean of quarterly (annual) growth stays around 0.95\% (3.8\%) until the first break 1965Q1 at which time an downward move to a mean quarterly (annual) growth rate to around $0.83 \%(3.2 \%)$ is shown. This level is preserved until the second break date around 1984Q1 at which time another negative shock decreases the mean quarterly (annual) growth rate to approximately $0.68 \%$ (2.7\%). We note that these estimates of growth are sensitive to the selection of the change point. Mean growth level at the beginning of the sample is much lower if observations prior to 1953 are disregarded. Furthermore, if the second change point is set later than 1984Q1, the decline in mean growth in the last period is more pronounced, hence it is important to assess break dates from data instead of this a priori definition ${ }^{1}$.

A second stylized feature that the data suggests is that the volatility of the real GDP growth series is changing in particular in the mid 1960s and mid 1980s. Basic findings regarding the volatility of the series are shown in Figure 1, which suggests that the volatilities in different sub-periods of the real GDP growth series is not the same, but show a declining behaviour in later years, especially after mid 1980s (the Great Moderation)2 Therefore, a second contribution would be adding the stochastic volatility assumption taking into account the fact that the volatility of the series is not constant, but changing over time.

\footnotetext{
${ }^{1}$ Given the range of their data, which spans the time period 1953Q2-1999Q2, McConnell and PerezQuiros (2000) conclude that the US real GDP series is stable. In our analysis, however, with an expanded data set, we reach a different conclusion.

${ }^{2}$ Indeed, for different sub-periods, we find that the sample standard deviations are 1.23,1.00 and 0.62, which are close to the volatility values reported in Blanchard and Simon (2001) using rolling estimation windows.
} 
Figure 1: Quarterly US GDP growth for the period between 1947Q1 and 2013Q4

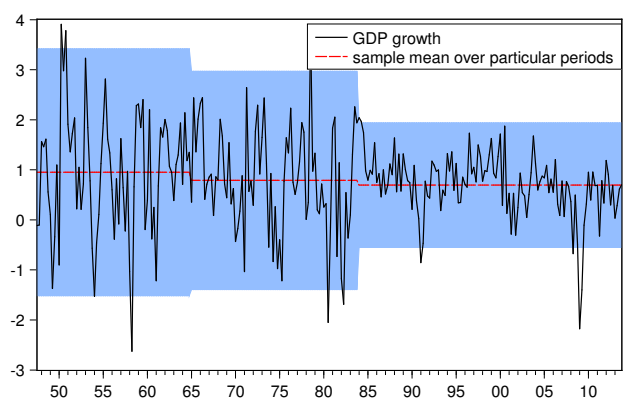

Note: The figure GDP growth data (in percentages), sample means over three sub-periods: 1947Q11964Q4, 1965Q1-1984Q4 and 1985Q1-2013Q4. The area bands are constructed as the area of two standard deviations around the sample mean, based on the sample standard deviation for each sub-periods.

Given that the information in macroeconomic series is usually not very strong about the typical data features of low and high frequencies, use is made of expectations data. These data are obtained from the Survey of Professional Forecasters (SPF), conducted by the Federal Reserve Bank of Philadelphia and are shown in Figure $2^{3}$. For the analysis, expected GDP growth for the next quarter is used for the period between 1969Q1 and 2013Q4. Prior to 1992, SPF data provides expected GNP growth. This difference in the survey results are corrected by adding the difference between GNP and GDP growth to data for each quarter before 1992. Given that these data follow the actual growth data reasonably well, in particular in recession periods, it may be expected that this information will strengthen the information in the likelihood function and as a consequence the empirical results.

\section{Basic model structures with time varying mean}

This section presents basic univariate models which allow for changes in the mean of the series. Possible identification issues are discussed in relation to relevant model parame-

\footnotetext{
${ }^{3}$ Expectations data is available from http://www.philadelphiafed.org/research-and-data/ real-time-center/survey-of-professional-forecasters/.
} 
Figure 2: One quarter ahead US GDP growth expectations from SPF for the period between 1969Q1 and 2013Q4

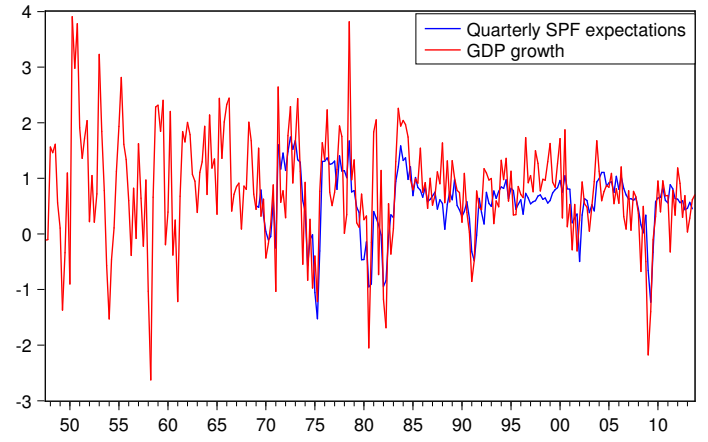

ters. The existence conditions for posterior distributions are analyzed under diffuse and informative priors.

In section 3.1, it is shown that time variation is not straightforward to incorporate even when a simple model for the data mean is considered. Identification and existence conditions need to be examined carefully. Specifically, one cannot completely free this standard model from assumptions on the long run expected mean: an intuitive and tractable as well as flexible structure must be assumed on the long run expected mean parameter. In section 3.2, we show that two alternative models, a simple local level model and the hierarchical linear mixed model, provide intuitive and flexible structures for the long run mean. We show that these alternative models have common properties in terms of identification, and a careful definition of priors enables Bayesian inference of these alternative models. We conclude, however, that these alternative models should be extended to incorporate macroeconomic data features of low and high frequencies by defining a realistic time series structure for the time varying means. 


\subsection{Basic models for time varying means}

Consider a simple model allowing for time varying means for a univariate time series $y_{t}$, $t=1, \ldots, T$ :

$$
y_{t}=\mu_{t}+\epsilon_{t}, \epsilon_{t} \sim \operatorname{NID}\left(0, \sigma_{\epsilon}^{2}\right),
$$

where $\mu_{t}$ is a scalar, time-varying parameter and $\sigma_{\epsilon}^{2}>0$.

Equation (1) is a general time-varying form of a standard model for the data mean, where the time variation in this mean, $\mu_{t}$, is not linked to any specific model or assumptions. As basic case one has $\mu_{t}=\mu, \forall t$ and this is the well known constant mean model. Given a flat prior the marginal posterior density of the constant mean, $\mu$, is proportional to the likelihood function and has the usual bell shaped curve with the posterior mean equal to the sample mean. This latter estimate is also used for forecasting. In this section, we consider different specifications for the time variation in means, and illustrate identification issues and existence conditions of the posterior for each specification.

The likelihood of the model in (1) is:

$$
\ell\left(y \mid \mu, \sigma_{\epsilon}^{2}\right)=\prod_{t=1}^{T} \ell\left(y_{t} \mid \mu_{t}, \sigma_{\epsilon}^{2}\right)=\prod_{t=1}^{T} \phi\left(y_{t} ; \mu_{t}, \sigma_{\epsilon}^{2}\right) \propto\left(\sigma_{\epsilon}^{2}\right)^{-T / 2} \prod_{t=1}^{T} \exp \left(-\frac{\left(y_{t}-\mu_{t}\right)^{2}}{2 \sigma_{\epsilon}^{2}}\right)
$$

where the $\phi\left(x ; \mu, \sigma^{2}\right)$ denotes the normal density function with mean $\mu$ and variance $\sigma^{2}$. In order to construct this likelihood, we make use of (1) which indicates that observations are independent of each other, given the model parameters, and that the observation at time $t$ depends on the time-invariant parameter $\sigma_{\epsilon}^{2}$ and the time varying parameter $\mu_{t}$ at time $t$. 
Non existence of the posterior in the general time-varying mean model under a flat prior

We show first that the model in (1) leads to an unbounded likelihood function without further restrictions on the time varying parameters $\mu_{t}$. For this purpose, consider the parameter points where $\mu_{t}=y_{t}, \forall t$ and let $\sigma_{\epsilon}^{2} \rightarrow 0$. The likelihood with these parameter settings is:

$$
\begin{aligned}
\lim _{\sigma_{\epsilon}^{2} \rightarrow 0} \ell\left(y \mid \mu=y, \sigma_{\epsilon}^{2}\right) & \propto \lim _{\sigma_{\epsilon}^{2} \rightarrow 0}\left(\left(\sigma_{\epsilon}^{2}\right)^{-T / 2} \prod_{t=1}^{T} \exp \left(-\frac{\left(y_{t}-\mu_{t}\right)^{2}}{2 \sigma_{\epsilon}^{2}}\right)\right) \\
& =\lim _{\sigma_{\epsilon}^{2} \rightarrow 0}\left(\left(\sigma_{\epsilon}^{2}\right)^{-T / 2}\left(\exp \left(-\frac{0}{2 \sigma_{\epsilon}^{2}}\right)\right)^{T}\right)=\infty .
\end{aligned}
$$

For a similar result for a model with time varying variances, we refer to De Pooter et al. (2009). Hence, under flat priors on the parameters, the posterior in (1) does not exist. The reason for this result is that we are in a situation of overfitting and lack of degrees of freedom: There are $T$ observations and one has $T+1$ parameters to estimate. A mechanical way to deal with this degree of freedom problem is to assume that one has several sub periods where the mean growth is constant, albeit different in each sub period, and that one knows a priori the switching points in the mean growth. Given at least three observations where the mean is constant, the posterior is proper under a flat prior, see also De Pooter et al. (2009). However, this is a too mechanical solution of the existence problem since in most cases one does not have such precise information on switch points. A different way of dealing with the existence issue is to impose a probability distribution for the time varying parameters, as in a local level model or a random coefficients model. In the remainder of this section, we show that such restrictions can be employed in a Bayesian setting with adequate prior distributions for the mean parameter. 


\subsection{Shapes of likelihood functions for alternative model struc- tures}

A Simple Local Level Model (SLLM) In order to impose identifying restrictions on the model in (1), we first consider a simple local level model for the time-varying means, $\mu_{t}$ such that:

$$
\begin{aligned}
& y_{t}=\mu_{t}+\epsilon_{t}, \quad \epsilon_{t} \sim \operatorname{NID}\left(0, \sigma_{\epsilon}^{2}\right), \\
& \mu_{t}=\mu^{\star}+\eta_{t}, \quad \eta_{t} \sim \operatorname{NID}\left(0, \sigma_{\eta}^{2}\right),
\end{aligned}
$$

where $\mu^{\star}$ is a scalar, $\sigma_{\epsilon}^{2}>0, \sigma_{\eta}^{2}>0$ and $E\left(\epsilon_{t}, \eta_{k}\right)=0, \forall t, k$.

The model in (5) and (6) imposes a shrinkage structure on the time varying parameters $\mu_{t}$ towards a constant value $\mu^{\star}$ and the posterior is proper but a new problem arises. The two variances of the disturbances defined in this model are not identified in this model under flat priors. This result is also known as a label switching problem, see FrühwirthSchnatter (2006). To show this, we obtain the reduced form model by inserting (6) in $(5)$ :

$$
y_{t}=\mu^{\star}+\epsilon_{t}+\eta_{t}=\mu^{\star}+v_{t}, \quad v_{t} \sim N I D\left(0, \sigma_{\epsilon}^{2}+\sigma_{\eta}^{2}\right),
$$

where the corresponding likelihood function is:

$$
\ell\left(y \mid \mu^{\star}, \sigma_{\epsilon}^{2}, \sigma_{\eta}^{2}\right)=\left(2 \pi\left(\sigma_{\epsilon}^{2}+\sigma_{\eta}^{2}\right)\right)^{-T / 2} \prod_{t=1}^{T} \exp \left(-\frac{\left(y_{t}-\mu^{\star}\right)^{2}}{2\left(\sigma_{\epsilon}^{2}+\sigma_{\eta}^{2}\right)}\right) .
$$

Assuming flat priors for $\mu^{\star}, \sigma_{\epsilon}^{2}$ and $\sigma_{\eta}^{2}$, the marginal posterior of the variance parame- 
ters can be calculated as follows:

$$
\begin{aligned}
p\left(\sigma_{\epsilon}^{2}, \sigma_{\eta}^{2} \mid y\right) & =\int p\left(\mu^{\star}, \sigma_{\epsilon}^{2}, \sigma_{\eta}^{2} \mid y\right) d \mu^{\star} \\
& \propto \int\left(\sigma_{\epsilon}^{2}+\sigma_{\eta}^{2}\right)^{-T / 2} \prod_{t=1}^{T} \exp \left(-\frac{\left(y_{t}-\mu^{\star}\right)^{2}}{2\left(\sigma_{\epsilon}^{2}+\sigma_{\eta}^{2}\right)}\right) d \mu^{\star} \\
& =\int\left(\sigma_{\epsilon}^{2}+\sigma_{\eta}^{2}\right)^{-T / 2} \prod_{t=1}^{T} \exp \left(-\frac{y_{t}^{2}-2 y_{t} \mu^{\star}+\left(\mu^{\star}\right)^{2}}{2\left(\sigma_{\epsilon}^{2}+\sigma_{\eta}^{2}\right)}\right) d \mu^{\star} \\
& =\int\left(\sigma_{\epsilon}^{2}+\sigma_{\eta}^{2}\right)^{-T / 2} \exp \left(-\frac{\sum y_{t}^{2}-2 T \bar{y} \mu^{\star}+T\left(\mu^{\star}\right)^{2}}{2\left(\sigma_{\epsilon}^{2}+\sigma_{\eta}^{2}\right)}\right) d \mu^{\star} \\
& =\left(\sigma_{\epsilon}^{2}+\sigma_{\eta}^{2}\right)^{-T / 2} \exp \left(-\frac{\sum y_{t}^{2}-T \bar{y}^{2}}{2\left(\sigma_{\epsilon}^{2}+\sigma_{\eta}^{2}\right)}\right) \int \exp \left(-\frac{T\left(\mu^{\star}-\bar{y}\right)^{2}}{2\left(\sigma_{\epsilon}^{2}+\sigma_{\eta}^{2}\right)}\right) d \mu^{\star}
\end{aligned}
$$

where the last equation shows that $\mu^{\star}$ has a conditional normal distribution with sample mean and variance $\left(\sigma_{\epsilon}^{2}+\sigma_{\eta}^{2}\right) / T$. Using this property, (13) becomes:

$$
p\left(\sigma_{\epsilon}^{2}, \sigma_{\eta}^{2} \mid y\right) \propto\left(\sigma_{\epsilon}^{2}+\sigma_{\eta}^{2}\right)^{-(T-1) / 2} \exp \left(-\frac{\sum_{t=1}^{T}\left(y_{t}-\bar{y}\right)^{2}}{2\left(\sigma_{\epsilon}^{2}+\sigma_{\eta}^{2}\right)}\right),
$$

i.e. the joint posterior is of an unknown form, the posterior of the sum of variance terms $\left(\sigma_{\epsilon}^{2}+\sigma_{\eta}^{2}\right)$ is an inverted Gamma density, and the conditional posteriors $p\left(\sigma_{\epsilon}^{2} \mid \sigma_{\eta}^{2}, y\right)$ and $p\left(\sigma_{\eta}^{2} \mid \sigma_{\epsilon}^{2}, y\right)$ are identical.

From (5) and (6), it can be seen that the conditional posteriors of the variance parameters are inverted Gamma densities, given flat priors for all model parameters. Similarly, the conditional posterior of $\mu^{\star}$ is a normal density from (6). Standard Gibbs sampling using these conditionals is possible despite the identification issue for the two variance parameters.

An illustration of the marginal posterior of the variance parameters in (14) is given in Figure 3 for simulated data with $T=10$ and $\left(\sigma_{\epsilon}^{2}+\sigma_{\eta}^{2}\right)=1$ for different values of $\sigma_{\epsilon}^{2}$ and $\sigma_{\eta}^{2}$ satisfying this restriction. Note that the posterior distribution exists under a flat prior on the parameters, but the two variance terms are not identified separately, hence the marginal posterior density has a ridge in Figure 3. 
Figure 3: Marginal posterior of the variance parameters for simulated data from the local level model in (5) and (6) for different values of $\sigma_{\epsilon}^{2}$ and $\sigma_{\eta}^{2}$ satisfying the restriction $\left(\sigma_{\epsilon}^{2}+\sigma_{\eta}^{2}\right)=1$.

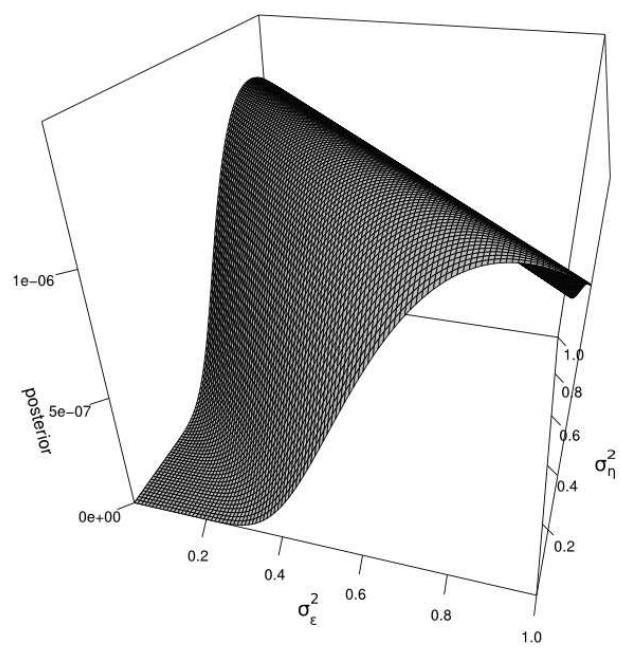

The identification or label switching problem in the SLLM can be avoided in several ways. A simple one is to impose the inequality condition that $\sigma_{\eta}^{2}$ is greater then $\sigma_{\epsilon}^{2}$ and to use proper prior distributions for $\sigma_{\epsilon}^{2}$ and $\sigma_{\eta}^{2}$. An alternative solution is to define a proper prior distribution on the so-called 'signal-to-noise ratio', defined by $q=\sigma_{\eta}^{2} / \sigma_{\epsilon}^{2}$. Rewriting the likelihood in (8) with this change of parameters we obtain:

$$
\ell\left(y \mid \mu^{\star}, \sigma_{\epsilon}^{2}, q\right)=\left(2 \pi(1+q) \sigma_{\epsilon}^{2}\right)^{-T / 2} \prod_{t=1}^{T} \exp \left(-\frac{\left(y_{t}-\mu^{\star}\right)^{2}}{2(1+q) \sigma_{\epsilon}^{2}}\right)
$$

A 'regularizing prior' for the model can be obtained, e.g. with a normal prior for the signal-to-noise ratio, $q \sim N_{[0, \infty)}\left(0, \sigma_{q}^{2}\right)$ and flat priors for $\mu^{\star}$ and $\sigma_{\epsilon}^{2}$. Combining the likelihood in (15) with these priors, the joint marginal posterior of the variance parameter and the signal-to-noise-ratio are:

$$
p\left(\sigma_{\epsilon}^{2}, q \mid y\right) \propto \int\left((1+q) \sigma_{\epsilon}^{2}\right)^{-T / 2}\left(\sigma_{q}^{2}\right)^{-1 / 2} \exp \left(-\frac{q^{2}}{2 \sigma_{q}^{2}}\right) \prod_{t=1}^{T} \exp \left(-\frac{\left(y_{t}-\mu^{\star}\right)^{2}}{2(1+q) \sigma_{\epsilon}^{2}}\right) d \mu^{\star}
$$

where the integration steps for $\mu^{\star}$ can be followed as in (9)-(13), and the marginal posterior 
Figure 4: Marginal posterior of the variance parameters for simulated data from the local level model in (5) and (6) for different values of $\sigma_{\epsilon}^{2}$ and $\sigma_{\eta}^{2}$, under the prior $q \sim N_{[0, \infty)}(0,0.5)$ and flat priors for the rest of the parameters.

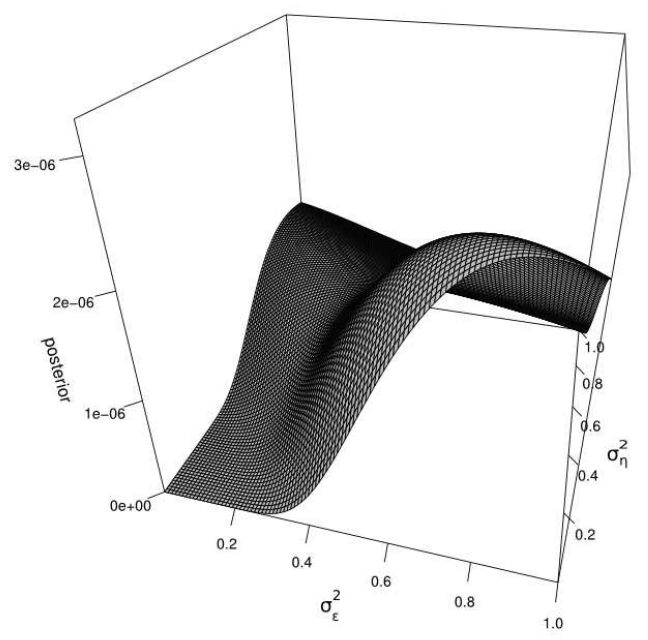

of the variance parameters is:

$$
p\left(\sigma_{\epsilon}^{2}, q \mid y\right) \propto\left((1+q) \sigma_{\epsilon}^{2}\right)^{-(T-1) / 2}\left(\sigma_{q}^{2}\right)^{-1 / 2} \exp \left(-q^{2} /\left(2 \sigma_{q}^{2}\right)\right) \prod_{t=1}^{T} \exp \left(-\frac{\left(y_{t}-\bar{y}\right)^{2}}{2(1+q) \sigma_{\epsilon}^{2}}\right)
$$

i.e. the regularizing prior only adds the factor $\exp \left(-q^{2} /\left(2 \sigma_{q}^{2}\right)\right)$ to the posterior density in (14). Figure 3 illustrates the marginal posterior density of $\sigma_{\epsilon}^{2}$ and $\sigma_{\eta}^{2}$ in (17) with $q \sim N_{[0, \infty)}(0,0.5)$ for simulated data with $T=10$ observations, where the marginal posterior has a clear peak and no ridge is visible. Notice that the definition of a prior for the signal-to-noise ratio relates to the properties of the SLLM. In the extreme case of $q=0$, there is no time variation in this model. On the other hand, when $q \rightarrow \infty$, all variation in the data are captured by the state equation disturbances. We emphasize that in the empirical analysis, we make use this ratio of variances in order to specify proper and regularizing priors for the variance parameters of the measurement and state equations.

A Hierarchical Linear Mixed Model (HLMM) An equivalent simplification of the general time varying parameter model in (1) is achieved by interpreting the SLLM as 
a random coefficients model that is known as the Hierarchical Linear Mixed Model, see for instance Hobert and Casella (1996). The HLMM defines the model restrictions by specifying priors for the time-varying parameters.

We illustrate the HLMM model by defining normal prior hyperparameters $\mu^{\star}$ and $\sigma_{\eta}^{2}$ and using a normal prior for the time-varying mean:

$$
\mu_{t} \mid \mu^{\star}, \sigma_{\eta}^{2} \sim N I D\left(\mu^{\star}, \sigma_{\eta}^{2}\right)
$$

It is easily seen that the HLMM model with the prior distribution in (18) and flat priors for the rest of the parameters is equivalent to the simple local level model in (5) and (6). Therefore, the identification issue, and the possibility of avoiding the identification issue using a proper prior for the signal-to-noise ratio are valid for this model as well.

Given the results on the basic models for time-varying means, the SLLM and HLMM, the next step is to incorporate data features which are more complex than the simple shrinkage mean considered so far. Specifically, incorporating macroeconomic data features of low and high frequencies should be incorporated in a meaningful and tractable way in

order to model the time series features of time varying means. In section 4 we present a set of alternative extended models and the corresponding prior distributions which aim to avoid the identification issue in the basic time-varying models.

\section{Prior and posteriors of extended model structures}

In this section, we present alternative models for US GDP growth, where different forms of time variation in long run growth are defined. In addition, the standard growth model is extended using data on growth expectations. The extended models are based on the conventional AR(1) model used for US GDP growth around a mean. 
This model is defined as follows

$$
\begin{aligned}
& y_{t}=\mu+v_{t} \quad t=1, \ldots, T \\
& v_{t}=\rho v_{t-1}+\epsilon_{t} \quad \epsilon_{t} \stackrel{i i d}{\sim} N\left(0, \sigma_{\epsilon_{t}}\right)
\end{aligned}
$$

where $y_{t}$ for $t=1, \ldots, T$ is the GDP growth at time $t, \rho$ is the autocorrelation coefficient, $\epsilon_{t}$ and $v_{t}$ are the disturbances and $\sigma_{\epsilon_{t}}>0$. The parameter $\mu$ in this model provides the expected long run growth rate.

Inserting (20) in (19), we obtain the following AR(1) model for GDP growth:

$$
y_{t}-\mu=\rho\left(y_{t-1}-\mu\right)+\epsilon_{t} \quad \epsilon_{t} \stackrel{i i d}{\sim} N\left(0, \sigma_{\epsilon_{t}}\right)
$$

which provides a model for growth in deviation from its expected mean. Note that when $\rho=1$ one has an identification problem since $\mu$ is not identified in this case. From the exploratory data analysis, it can be seen that this event is unlikely for this dataset. Otherwise, the approach of Schotman and van Dijk (1991b) and Schotman and van Dijk (1991a) can be used.

A straightforward extension of this model, which allows for time-variation in the longrun means is the well known local level model time series model with $\mu_{t}=\mu_{t-1}+\eta_{t}$, see Harvey (1989, ch. 2). Despite its general specification in terms of time-varying means, this local level model implies an I(1) process for growth data, which is unlikely to hold. Therefore, we propose alternative model structures with different time variation in parameters that do not assume an I(1) process for growth. 


\subsection{Level shifts}

The first extension of the model in (21), denoted by 'LS', introduces changes in long-run growth through 'occasional' shifts over time:

$$
\begin{aligned}
y_{t}-\mu_{t} & =\rho\left(y_{t-1}-\mu_{t-1}\right)+\epsilon_{t} \\
\mu_{t} & =\mu_{t-1}+\kappa_{t} \eta_{1, t}
\end{aligned}
$$

for $t=1, \ldots, T$ observations where $\mu_{t}$ is the time-varying long run growth, with initial value $\mu_{0} \sim N\left(\hat{\mu}_{0}, \sigma_{\mu_{0}}\right), \kappa_{t}$ has a Binomial distribution with parameter $p_{\kappa}, \epsilon_{t} \sim N I D\left(0, \sigma_{\epsilon}^{2}\right)$, $\eta_{1, t} \sim N I D\left(0, \sigma_{\eta_{1}}^{2}\right)$ and $E\left(\epsilon_{t}, \eta_{1, k}\right)=0, \forall t, k$. In this model, changes in levels depend on $p_{\kappa}$, hence only a single additional parameter is introduced in the model. Occasional and large level shifts correspond to low values of $p_{\kappa}$ together with high values of $\sigma_{\eta_{1}}$. When $p_{\kappa}$ is 1 , the model becomes standard local level model.

\subsection{Stochastic volatility}

The second extension of the model in (21), denoted by 'SV', introduces time variation in the volatility of growth through a stochastic volatility component in the observation equation in (22). For this extension we define time-varying variances such that $\epsilon_{t} \sim$ $N\left(0, \sigma_{\epsilon_{t}}^{2}\right)$ with $\sigma_{\epsilon_{t}}=\exp \left(h_{t} / 2\right)$, and the time varying stochastic volatility component is incorporated in the model through an additional state equation:

$$
h_{t}=h_{t-1}+\eta_{2, t}
$$

where $\eta_{2, t} \sim N I D\left(0, \sigma_{\eta_{2}}^{2}\right)$. Note that parameter $\sigma_{\eta_{2}}^{2}$ can be estimated from data, but the empirical identification of this variance parameter is not trivial. 


\subsection{Survey data on growth expectations}

The final extension to the standard growth model is the inclusion of growth expectations data, denoted by 'EXP', as an explanatory variable in the model. Since the growth model is defined in deviations from (possibly time-varying) means, we consider a model specification where the growth expectations are also modeled in deviation from expected means. For this purpose, the observation equation in the generalized model in (22) is rewritten as follows:

$$
y_{t}-\mu_{t}=\rho\left(y_{t-1}-\mu_{t-1}\right)+\beta\left(S_{t}-\mu_{t}\right)+\epsilon_{t}, \epsilon_{t} \sim N\left(0, \sigma_{\epsilon_{t}}^{2}\right),
$$

where $S_{t}$ denotes the expected growth from survey data for time $t$, obtained in the previous period $t-1$. In this model, mean growth can be defined as a constant mean or as the level shift specification in (23). Similarly, a constant volatility or SV model in (24) can be adopted depending on the definition of $\sigma_{\epsilon_{t}}^{2}$.

\subsection{Bayesian inference for proposed models}

Given the above alternative model extensions, conventional priors can be used for each model parameter and posterior samplers are based on Gibbs sampling for the parameters.

The conventional priors refer to normal or flat priors for the mean $\mu$ or the autocorrelation coefficient $\rho$, and inverse gamma priors for the variance parameters. The conditional posterior densities of these parameters then follow normal and inverse gamma distributions.

For the models with level shifts, conditional on other model parameters and level shifts $\kappa_{t}$, draws from unobserved states are obtained using the Kalman Filtering and Smoothing algorithm, see Carter and Kohn (1994) and Frühwirth-Schnatter (1994). Conditional on the unobserved states, draws from level shifts are obtained using the algorithm proposed by Gerlach et al. (2000), see also Giordani and Kohn (2008) for details. 
Finally, for models with a stochastic volatility component, draws from the unobserved variable $h_{t}$ can be obtained using the Kalman filter and smoother, similar to the unobserved states $\mu_{t}$. However, since the SV model is based on the logarithmic transformation of the variance, the resulting conditional density follows a log- $\chi^{2}$ distribution. Noticing the properties of $\log -\chi^{2}$ distribution, Kim et al. (1998) and Omori et al. (2007) approximate this distribution using a mixture of Gaussian distributions. Hence, conditional on these mixture components the system remains Gaussian allowing for standard inference outlined above. For details, see Omori et al. (2007).

For a detailed description of the all the sampling steps, we refer to the Appendix and to(Baştürk et al., 2014).

\section{Empirical results}

In this section we apply the standard and extended growth models to quarterly US GDP growth for the period between 1947Q1 and 2013Q4. As described in section 4, five different model structures are considered US GDP data:

AR autocorreltion/serial correlation in errors

LL local level model for GDP growth levels, i.e. GDP growth level is subject to continuous changes over time

LS level shifts in GDP growth, i.e. GDP growth level is subject to occasional changes over time

SV stochastic volatility in GDP growth, i.e. GDP growth volatility changes over time

EXP growth model incorporating growth expectations from professional forecasters as an additional explanatory variable

where one or more of these structures are combined in alternative models. 


\subsection{Prior definitions}

The priors for the parameters of alternative model structures are defined independently, as outlined in section 4. For models with serial correlation (AR), a flat prior is defined for the correlation coefficient: $\rho \sim U(0,1)$. For standard growth models with no changes in GDP growth level, a flat prior is defined for mean GDP growth $\mu \sim U(-\infty, \infty)$. For models incorporating GDP growth expectations, a flat prior is defined for the effect of expectations, i.e. $\beta \sim U(-\infty, \infty)$.

For models with constant error variance for GDP growth variance, we define proper but uninformative priors: $\sigma_{\epsilon}^{2} \sim I G\left(T / 2, \sigma^{2} / 2 \times T / 2\right)$, where $I G(a, b)$ denotes the inverse Gamma distribution with shape $a$ and scale $b, T=267$ is the number of observations and $\sigma^{2}$ is the data variance. This data based prior implies that the prior is equivalent to $T / 2$ observations, hence we expect the data with $T$ observations to dominate the prior.

In addition, for models with level changes in GDP growth (LL, LS), we define inverse Gamma priors: $\sigma_{v}^{2} \sim I G\left(T / 2, \sigma^{2} / 20 \times T / 2\right)$. Note that the a priori mean of this variance is 10 times smaller than the a priori mean of the observation variance defined above. This specification is based on the intuition that GDP growth levels, if modeled as time varying components, should not have drastic changes over time but capture longer run GDP growth levels. Despite this definition, the prior we define is still relatively uninformative compared to the information in the likelihood: it is equivalent to $T / 2$ observations only and the data information is likely to dominate this prior.

For models with occasional level changes in GDP growth (LS) we set a prior level change probability of 0.04 , indicating around 5 a priori expected changes in gdp levels during the sample period. For models with the stochastic volatility component and occasional level changes in GDP growth, we set a prior level change probability of 0.02 . This lower prior probability for a level change is intended to empirically identify more accurately the mean and variance changes at the same time. 
Table 1: Estimation results from alternative models

\begin{tabular}{|c|c|c|c|c|}
\hline model & $\mu$ & $\rho$ & $\overline{\sigma_{\epsilon}^{2}}$ & $\overline{\sigma_{v}^{2}}$ \\
\hline \multicolumn{5}{|c|}{ models without level changes } \\
\hline \multirow[t]{2}{*}{ constant mean } & 0.793 & - & 0.885 & - \\
\hline & $(0.059)$ & - & $(0.072)$ & - \\
\hline \multirow[t]{2}{*}{$\mathrm{AR}$} & 0.792 & 0.380 & 0.775 & - \\
\hline & $(0.089)$ & $(0.056)$ & $(0.064)$ & - \\
\hline \multirow[t]{2}{*}{ AR-SV* } & 0.804 & 0.377 & 0.356 & - \\
\hline & $(0.044)$ & $(0.056)$ & $(0.241)$ & - \\
\hline \multicolumn{5}{|c|}{ models with frequent level changes } \\
\hline \multirow[t]{2}{*}{ LL } & - & - & 0.569 & 0.054 \\
\hline & - & - & $(0.038)$ & $(0.006)$ \\
\hline \multirow[t]{2}{*}{ AR-LL } & - & 0.251 & 0.562 & 0.087 \\
\hline & - & $(0.077)$ & $(0.042)$ & $(0.034)$ \\
\hline \multicolumn{5}{|c|}{ models with occasional level changes } \\
\hline \multirow[t]{2}{*}{$\mathrm{LS}$} & - & - & 0.743 & 0.024 \\
\hline & - & - & $(0.055)$ & $(0.003)$ \\
\hline \multirow[t]{2}{*}{ AR-LS } & - & 0.368 & 0.685 & 0.023 \\
\hline & - & $(0.055)$ & $(0.049)$ & $(0.003)$ \\
\hline \multirow[t]{2}{*}{ AR-LS-SV* } & - & 0.321 & 0.305 & 0.064 \\
\hline & - & $(0.078)$ & $(0.238)$ & $(0.012)$ \\
\hline
\end{tabular}

Note: The table reports posterior means and standard deviations (in parentheses) of model parameters. $\sigma_{\epsilon}^{2}$ and $\sigma_{v}^{2}$ are the observation and state variances, respectively. $(*)$ indicates that the observation variance is time varying in these models. For these models we report mean observation variance and standard deviation for the sample period. Results are based on 10000 draws of which 5000 are burn-in draws.

\subsection{Estimation results}

In this subsection, we report estimation results from eight alternative models combining one or more of the AR, SV, LL and LS model structures. ${ }^{4}$ Table 1 presents parameter estimates from all compared models based on 10000 draws where we consider the first 5000 draws as burn-in draws.

Despite the differences in model structures, Table 1 shows that parameter estimates from alternative models are similar across all models we consider. For models with con-

\footnotetext{
${ }^{4}$ Extended models incorporating growth expectations are analyzed separately since the sample size is much smaller due to the range of the data on growth expectations.
} 
stant mean, mean quarterly growth is around 0.8 , which is in line with the visual inspection of the data. For models with serial correlation, the posterior mean for the correlation coefficient is around 0.3, i.e. correlation levels seem to be far from the nonidentification region $\rho=1$. Two unique models in these alternative models are LL and AR-LL models, which imply a unit root in GDP growth. Despite this counter-intuitive assumption, results of the LL and AR-LL models are roughly in line with the constant mean models in terms of parameter estimates.

Table 1 shows that models which allow for time variation in means, with LL and LS components, in general lead to smaller observation variances compared to the constant mean models (AR and AR-SV). This result is intuitive since part of the data variation is explained by the changing means in models with the time variation in mean growth. Furthermore, estimated state variances are very small compared to the observation variance, indicating rather smooth changes in growth levels over time.

We next report estimated growth means for the five models with time variation in means, namely LL, AR-LL, LS, AR-LS and AR-LS-SV models. Figure 5 presents these estimates together with the $95 \%$ HPDI for estimated mean growth. All models imply changing growth means over time. In line with the model definitions, AR-LL and LL models lead to more volatile growth levels over time while AR-LS and AR-LS-SV models lead to less volatile growth levels. All models imply relatively high growth levels at the beginning of the sample and relatively low growth levels towards the end of the sample. These results confirm stylized facts reported in section 2 although we find more than two periods of level changes in AR-LS and AR-LS-SV models, as shown in Figure 6.

Finally, we note that incorporating the stochastic volatility component in the model seems to be important. Estimated volatility levels from the two models with SV component, AR-SV and AR-LS-SV, are given in Figure 7. Both models indicate substantial changes in volatility over time. Estimated volatility also confirms the stylized facts covered in section 2: the period of the 50s and the beginning of the 60s are characterized by 
Figure 5: GDP growth level estimates from alternative models with time varying growth means
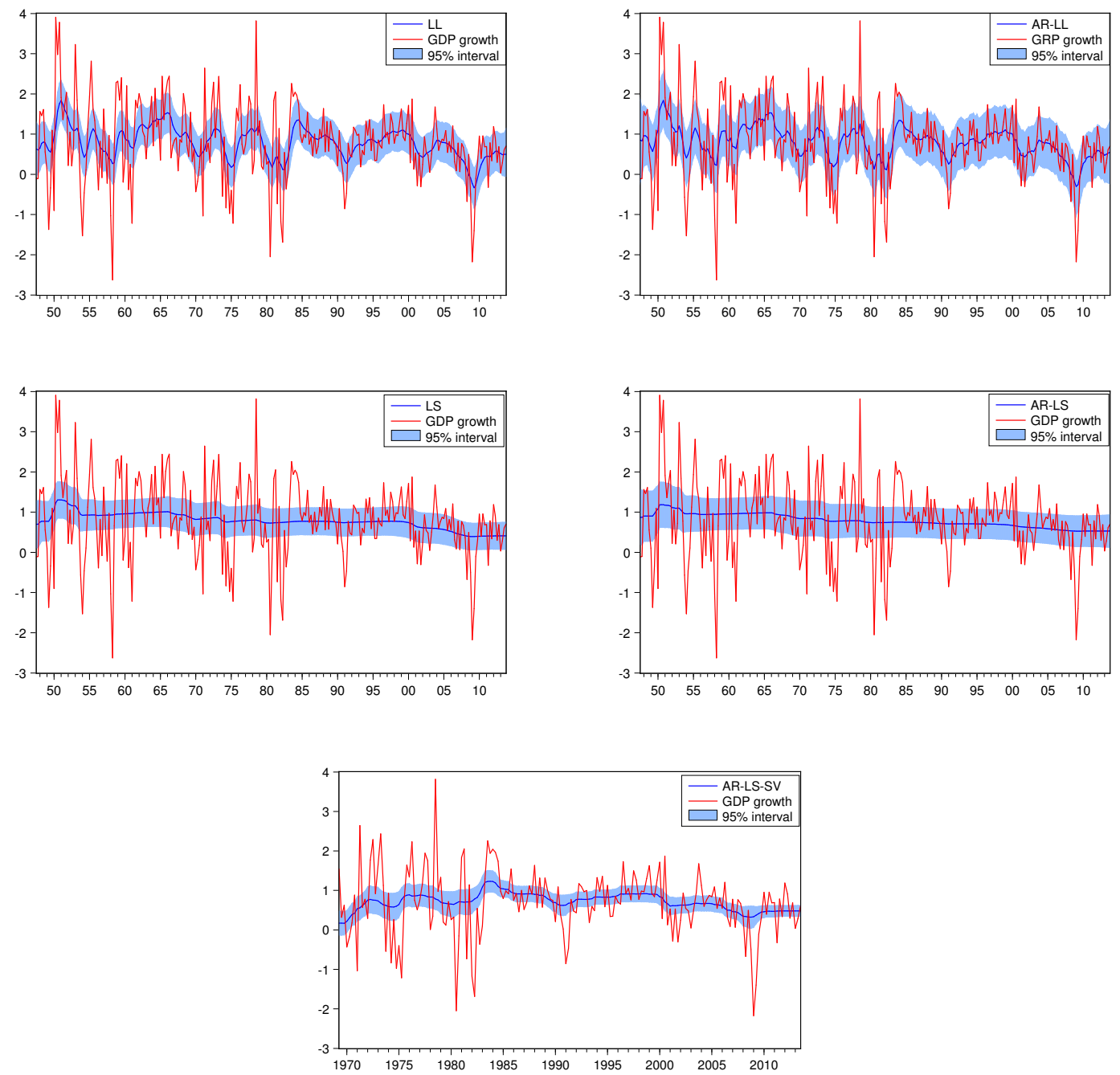

Note: The figure presents mean posterior levels for GDP growth $(\times 100)$ for compared models for the whole sample period. Results are based on 10000 draws of which 5000 are burn-in draws. 
Figure 6: Estimated level shifts for models with occasional level shifts (AR-LS and ARLS-SV)
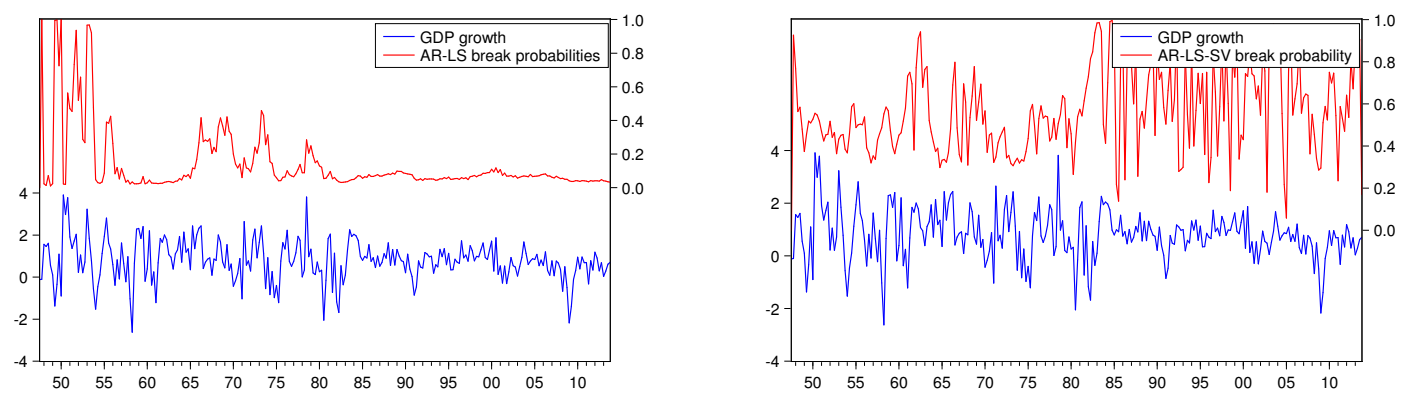

Figure 7: Estimated mean volatility for models with stochastic volatility (AR-SV and AR-LS-SV)
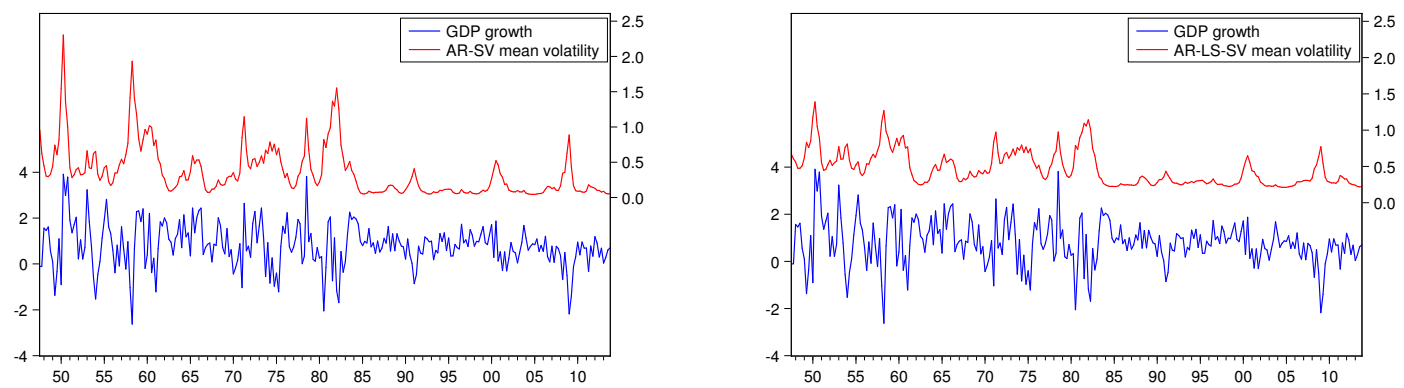

relatively high volatility in growth and the volatility level decreases towards the end of the sample period, apart from the recession of 2000 and the recent crisis. Comparing the two panels in Figure 7, it can be seen that allowing for time variation in means (AR-LS-SV) leads to much lower volatility levels compared to the counterpart model with constant means (AR-SV) at the beginning of the sample. In other words, the volatile period of 50s is explained both by the changing means and changing volatility in AR-LS-SV model.

\subsection{Prediction results}

In order to assess the predictive performance of the compared models in section 5.2, we consider 1 quarter ahead forecasts of GDP growth for the period between 1997Q1 and 2013Q4. We compare the predictive performance of models using the average MSFE 
Table 2: 1 period ahead MSFE and cumulative predictive likelihoods

\begin{tabular}{lcc}
\hline model & MSFE & Cumulative Pred. Lik. \\
\cline { 1 - 2 } models without level changes & \\
constant mean & 0.5170 & -76.2513 \\
AR & 0.3916 & -69.4748 \\
AR-SV & 0.3990 & -72.6564 \\
models with frequent level changes \\
LL & 0.5347 & -72.6307 \\
AR-LL & 0.5035 & -70.9595 \\
models with occasional level changes \\
LS & 0.4748 & -70.9052 \\
AR-LS & 0.3905 & -66.0502 \\
AR-LS-SV & 0.3888 & -57.7819 \\
\hline
\end{tabular}

and cumulative predictive likelihood for the prediction period. These results are given in Table 2 and Figure 8.

Table 2 and Figure 8 show that predictive performances of alternative models are quite different, despite the similar parameter estimates reported in section 5.2.

First, the serial correlation component is found to be important for the predictive performance of growth models since the standard AR model, the AR-LL and AR-LS models perform better in terms of MSFE and predictive likelihoods compared to their counterparts without the AR component, namely the constant mean model, LL model and LS model.

Second, models with frequent level shifts, the LL and AR-LL models, do not perform well compared to models with occasional level shifts, LS and AR-LS, in terms of the predictive likelihoods. According to these predictive performances, models allowing for occasional level shifts, i.e. slowly varying long-run growth levels, are more appropriate to forecast growth.

Third, models with occasional level shifts (AR-LS and AR-LS-SV) perform better than the standard growth model with serial correlation, hence changes in growth levels should 
Figure 8: Cumulative predictive likelihoods for the prediction sample from alternative models

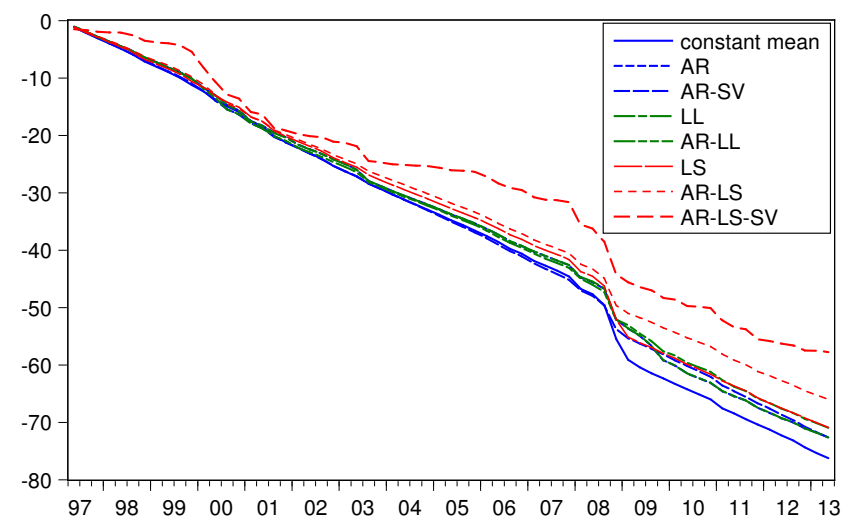

definitely be accounted for.

Finally, A comparison of the prediction results for AR-LS and AR-LS-SV models in Table 8 indicate that adding a stochastic volatility component improves the predictive performance of the growth model substantially, particularly according to the predictive likelihoods.

From these results, we conclude that the most appropriate model for predicting growth is the AR-LS-SV model, which takes into account the slowly changing levels of growth, serial correlation in disturbances and changing disturbance variances over time.

\subsection{Prior predictive likelihood analysis}

The predictive performance comparisons in section 5.3 are naturally affected by the prior choices for parameters, especially since the existence and identification issues illustrated in section 3 require proper priors for part of the model parameters in the alternative models we consider. In this subsection, we report the effect of the selected priors on prediction results. An important point is whether the superior performance of the AR-LS-SV can be attributed solely to prior definitions.

The effect of the priors are analyzed using a prior-predictive likelihood analysis. For 
Table 3: Prior predictive analysis: 1 period ahead MSFE and cumulative predictive likelihoods

\begin{tabular}{lcc}
\hline model & MSFE & Cumulative Pred. Lik. \\
\hline constant mean & 34.131 & -2297.532 \\
AR & 11.696 & -813.025 \\
AR-SV & 0.482 & -58.386 \\
models with frequent level changes \\
LL & 0.495 & -70.142 \\
AR-LL & 0.606 & -77.464 \\
models with & occasional level changes \\
LS & 0.499 & -66.682 \\
AR-LS & 0.503 & -62.260 \\
AR-LS-SV & 0.486 & -58.141 \\
\hline
\end{tabular}

this purpose, we do not update model parameters using data, but instead use parameter draws from prior densities, i.e. Gibbs sampling steps are performed for each model without updating the model parameters. For models with level changes and stochastic volatility, unobserved levels are updated using the data since these variables are not model parameters but unobserved states. For all considered models, MSFE and cumulative predictive likelihoods resulting from the prior-predictive likelihood analysis are presented in Table 3.

According to the prior-predictive analysis in Table 3, the AR-SV model is the 'best' alternative model in comparison to the preferred model, AR-LS-SV. MSFE and predictive likelihoods of these models are very similar without the likelihood information. Hence the superior predictive performance of the AR-LS-SV model in section 5.3 is not solely attributed to the prior definitions.

The 'least preferred' models according to the prior-predictive likelihoods are the constant mean model and the standard AR model. This result is expected for two reasons. First, uninformative prior densities for these models lead to very wide prediction intervals. $^{5}$ Second, in models with level changes and stochastic volatility, unobserved states

\footnotetext{
${ }^{5}$ One important point is the flat prior we use for the standard growth models with no change in
} 
are updated using the data even though model parameters are not updated. Hence these models make use of data information to update unobserved states regardless of the prior definition.

Table 3 also shows that the models with occasional level shifts (LS, AR-LS, AR-LSSV) are not necessarily favored by the prior as the AR-SV model, without mean changes, leads to better prior-predictive results than most models with occasional level shifts. The conclusion on the necessity to include occasional level shifts in section 5.3 does not seem to be the result of the employed prior densities. Similarly, models with AR components are also not necessarily favored by the prior definitions.

Comparing the AR-LS and AR-LS-SV models in Table 3, we conclude that the priors favor the model stochastic volatility component. Despite this result, the prior domination seems to be less than the data domination particularly for predictive likelihoods: The improvement in predictive likelihoods of the AR-LS-SV model is much more pronounced in Table 2 compared to the prior-predictive likelihood comparison in Table 3. Furthermore, the priors favor the simple AR-SV model substantially, according to the MSFE and cumulative predictive likelihoods. Despite this finding, the preferred model, AR-LS-SV, is found to perform better than the simple AR-SV model in the predictive analysis in section 5.3.

\subsection{Results using growth expectations}

In this subsection, we consider growth models incorporating growth expectations as a final addition to the proposed growth models. We note that the expectation data is only available for the period between 1969Q1 and 2013Q4. Therefore, for a fair comparison of models, alternative models are estimated for this smaller sample period. Similarly, prediction comparisons, in terms of MSFE and predictive likelihoods, are also based on the time period for which expectation data is available.

levels. For the prior predictive analysis, we truncate this prior density between $[-10,10]$ since none of the posterior draws lie outside this region. 
Table 4: Estimation results from alternative models for US growth between 1969Q1 and $2013 \mathrm{Q} 3$

\begin{tabular}{|c|c|c|c|c|c|}
\hline model & $\mu$ & $\rho$ & $\beta$ & $\overline{\sigma_{\epsilon}^{2}}$ & $\overline{\sigma_{v}^{2}}$ \\
\hline \multicolumn{6}{|c|}{ models without level changes } \\
\hline \multirow[t]{2}{*}{$\mathrm{AR}$} & 0.804 & 0.377 & - & 0.356 & - \\
\hline & $(0.044)$ & $(0.056)$ & - & $(0.241)$ & - \\
\hline \multirow[t]{2}{*}{ AR-EXP } & 0.679 & 0.131 & 0.456 & 0.570 & - \\
\hline & $(0.488)$ & $(0.079)$ & $(0.133)$ & $(0.059)$ & - \\
\hline \multicolumn{6}{|c|}{ models with occasional level changes } \\
\hline \multirow[t]{2}{*}{ AR-LS-SV* } & - & 0.280 & - & 0.294 & 0.036 \\
\hline & - & $(0.097)$ & - & $(0.144)$ & $(0.006)$ \\
\hline \multirow[t]{2}{*}{ AR-LS-SV-EXP* } & - & 0.118 & 0.106 & 0.288 & 0.035 \\
\hline & - & $(0.083)$ & $(0.039)$ & $(0.138)$ & $(0.005)$ \\
\hline
\end{tabular}

Note: For all models, sample is determined as the period for which expectation data is available. Results are based on 10000 draws of which 5000 are burn-in draws.

Table 4 presents estimation results for 4 models, the constant mean, AR, AR-EXP, AR-LS-SV and AR-LS-SV-EXP models. For both models with expectations, AR-EXP and AR-LS-SV-EXP, 95\% HPDI for parameter $\beta$ is found to be on the positive region, i.e. expectations are found to drive GDP growth (in deviation from the long-run mean). Furthermore, posterior means for the serial correlation parameter, $\rho$, is lower when expectations are incorporated in the models hence part of the persistence in growth rates are captured by the persistence in expectations.

Figure 9 presents estimated levels, break probabilities and mean volatilities for ARLS-SV and AR-LS-SV-EXP models, i.e. models with time varying levels. Comparing the graphs top panel in Figure 9, it can be seen that including growth expectations in the model leads to smoother estimated long-run levels since part of the variation in growth is captured by the variation in growth expectations. Despite this finding, the middle and bottom panels in Figure 9 shows that estimate break probabilities and volatility of growth follow similar patterns regardless of the inclusion of expectations in the model.

For a detailed comparison of the models with and without growth expectations, we 
Figure 9: Estimation results for the AR-LS-SV model and AR-LS-SV-EXP models
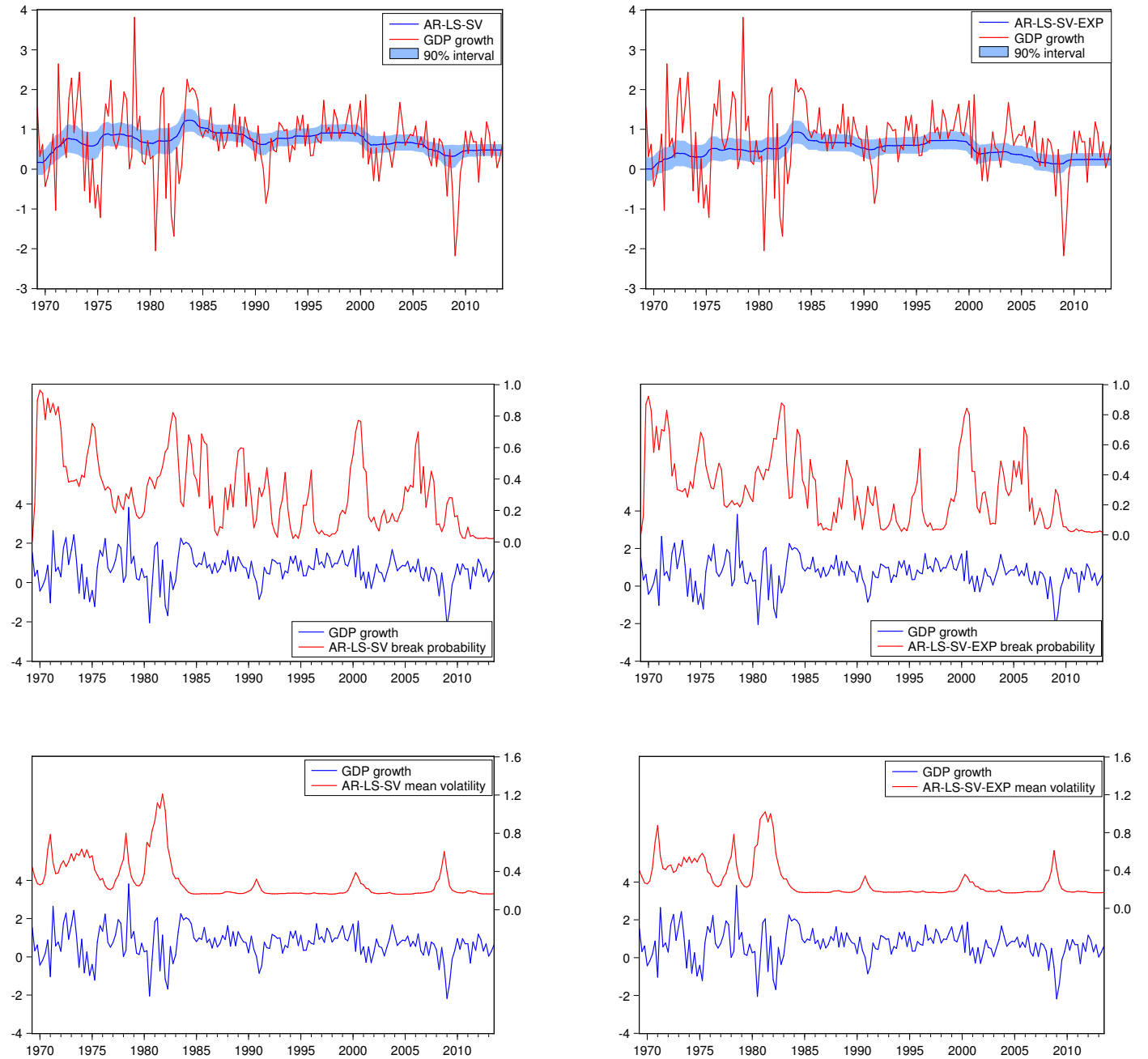

Note: The figure presents estimated levels, break probabilities and mean volatility for GDP growth (in percentages) for the sample period 1969Q2-2013Q4, the period for which growth expectations are available. Results are based on 10000 draws of which 5000 are burn-in draws. 
Table 5: Prediction results from alternative models for US growth between 1969Q1 and 2013Q3

\begin{tabular}{lcc}
\hline model & MSFE & Cumulative Pred. Lik. \\
\hline models without level changes & \\
AR & 0.360 & -76.78 \\
AR-EXP & 0.367 & -77.52 \\
models with occasional level changes \\
AR-LS-SV & 0.371 & -65.16 \\
AR-LS-SV-EXP & 0.375 & -64.54 \\
\hline
\end{tabular}

Note: For all models, sample is determined as the period for which expectation data is available. Forecast sample includes data between 1994Q4 and 2013Q4. Results are based on 10000 draws of which 5000 are burn-in draws.

consider the predictive performances of the four models in Table 4, using 1 quarter ahead MSFE and cumulative predictive likelihoods. The predictive performances are reported in Table 5 for the forecast period 1994Q4 and 2013Q4.

The results in Table 5, which are based on a smaller sample period compared to section 5.3, confirm that modeling occasional shifts (AR-LS-SV or AR-LS-SV-EXP) in mean growth improve the predictive power of standard growth models. Secondly, according to the MSFE values reported in Table 5, adding expectations to AR or AR-LS models causes a slight deterioration point forecasts. However, according to the cumulative predictive likelihoods, expectations clearly improve predictive power of these models. Hence, if the purpose is to obtain density forecasts of growth, incorporating expectations in the model structure is important.

The evolution of predictive likelihoods for all compared models through the forecast sample period are shown in Figure 10. The model incorporating expectations, AR-LSSV-EXP, leads to improvements in predictive likelihoods in two important periods in the sample: the period between 2000 and 2004, and between 2008 and 2009, i.e. the recession in early 2000s and the recent Great recession. We therefore conclude that incorporating expectations in growth models can be useful especially in turbulent times, even if the 
Figure 10: Cumulative predictive likelihoods from alternative models for US growth between 1969Q1 and 2013Q3

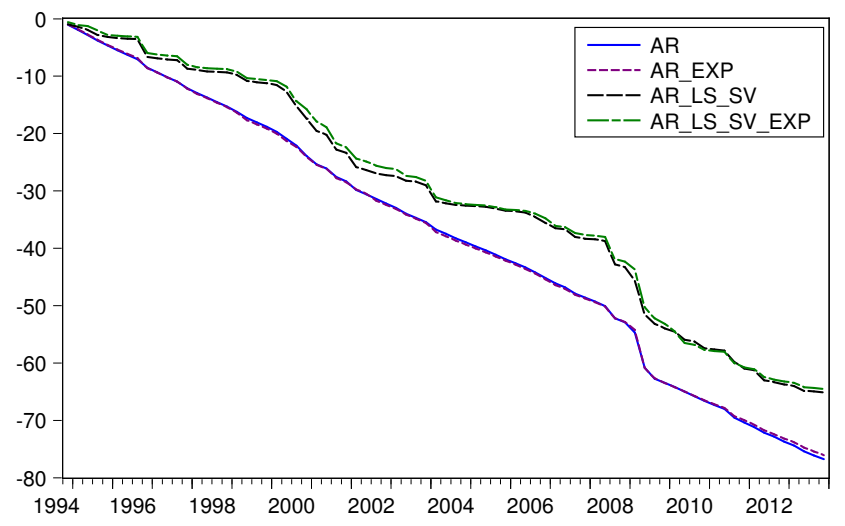

Note: For all models, sample is determined as the period for which expectation data is available. Forecast sample includes data between 1994Q4 and 2013Q4. Results are based on 10000 draws of which 5000 are burn-in draws.

performance of the models point forecasts (measured by MSFE) does not improve with the addition of expectation data.

\section{Final remarks}

We analyzed time varying patterns in US growth using various univariate model structures, starting from a naive model structure where all features change every period to a model where the slow variation in the conditional mean and changes in the conditional variance are specified together with their interaction, including survey data on expected growth in order to strengthen the information in the model. Our results indicate that incorporating time variation in mean growth rates as well as volatility of mean growth rates are important for improving the predictive performances of standard growth models. Furthermore, using data information on growth expectations is important for forecasting growth in specific periods, such as the 2000s and around 2008.

The empirical analysis leads to several topics for further research in relation to in- 
corporating time varying patterns and growth expectations in growth models. First, the approach of this paper can be extended to analyze the difference between actual and potential growth, the so-called growth gap and also to analyze features of the business cycle like the dating procedure of Pagan and Harding (2005). Second, instead of 'selecting' a specific model, the proposed models with time variation or with GDP expectations can be 'combined', as in Billio et al. (2013) to combine the weights of the expectations data and the model information over time, with possibly an improvement of the overall predictive performance of the growth model. Finally, the proposed models can be applied to GDP growth of countries other than the US in order to analyze international evidence on the predictive gains from changing growth levels, variances and incorporating growth expectations in standard growth models. This requires extending our work and including a panel data model. 


\section{References}

Baştürk N, Çakmaklı C, Ceyhan P, Van Dijk HK. 2014. Posterior-predictive evidence on US inflation using extended Phillips Curve models with non-filtered data. Forthcoming in Journal of Applied Econometrics.

Billio M, Casarin R, Ravazzolo F, Van Dijk HK. 2013. Time-varying combinations of predictive densities using nonlinear filtering. Journal of Econometrics 177: 213-232. ISSN 0304-4076. Dynamic Econometric Modeling and Forecasting.

Blanchard O, Simon J. 2001. The long and large decline in U.S. output volatility. Brookings Papers on Economic Activity 32: 135-174.

URL http://ideas.repec.org/a/bin/bpeajo/v32y2001i2001-1p135-174.html

Carter CK, Kohn R. 1994. On Gibbs sampling for state space models. Biometrika 81: $541-553$.

Clarida R, Galí J, Gertler M. 2000. Monetary policy rules and macroeconomic stability: Evidence and some theory. The Quarterly Journal of Economics 115: 147-180. URL http://ideas.repec.org/a/tpr/qjecon/v115y2000i1p147-180.html

De Pooter M, Ravazzolo F, Segers R, Van Dijk HK. 2009. Bayesian near-boundary analysis in basic macroeconomic time-series models. In Chib S, Koop G, Griffiths W, Terrell D (eds.) Advances in Econometrics, (Bayesian Econometrics), volume 23. Emerald Group Publishing Limited, 331-402.

Frühwirth-Schnatter S. 1994. Data augmentation and dynamic linear models. Journal of Time Series Analysis 15: 183-202.

Frühwirth-Schnatter S. 2006. Finite Mixture and Markov Switching Models: Modeling and Applications to Random Processes. Springer Series in Statistics. Springer. 
Gerlach R, Carter C, Kohn R. 2000. Efficient Bayesian inference for dynamic mixture models. Journal of the American Statistical Association : 819-828.

Giordani P, Kohn R. 2008. Efficient Bayesian inference for multiple change-point and mixture innovation models. Journal of Business \& Economic Statistics 26: 66-77.

Harvey AC. 1989. Forecasting, structural time series models and the Kalman filter. Cambridge University Press.

Hildreth C. 1963. Bayesian statisticians and remote clients. Econometrica 31: 422-438.

Hobert JP, Casella G. 1996. The effect of improper priors on Gibbs sampling in hierarchical linear mixed models. Journal of the American Statistical Association 91: 1461-1473.

Kim CJ, Nelson CR. 1999. Has the u.s. economy become more stable? a Bayesian approach based on a markov-switching model of the business cycle. The Review of Economics and Statistics 81: pp. 608-616. ISSN 00346535.

URL http://www.jstor.org/stable/2646710

Kim CJ, Nelson CR, Piger J. 2004. The less-volatile US economy: a Bayesian investigation of timing, breadth, and potential explanations. Journal of Business $\mathcal{E}$ Economic Statistics 22: 80-93.

Kim S, Shephard N, Chib S. 1998. Stochastic volatility: Likelihood inference and comparison with ARCH models. Review of Economic Studies 65: 361-393.

McConnell MM, Perez-Quiros G. 2000. Output fluctuations in the united states: What has changed since the early 1980's? The American Economic Review 90: pp. 14641476. ISSN 00028282.

URL http://www.jstor.org/stable/2677860

Milani F. 2011. Expectation shocks and learning as drivers of the business cycle. Economic Journal 121: 379-401. 
URL http://EconPapers.repec.org/RePEc:ecj:econjl:v:121:y:2011:i:552:p: $379-401$

Omori Y, Chib S, Shephard N, Nakajima J. 2007. Stochastic volatility with leverage: Fast and efficient likelihood inference. Journal of Econometrics 140: 425-449.

Pagan AR, Harding D. 2005. A suggested framework for classifying the modes of cycle research. Journal of Applied Econometrics 20: 151-159.

URL http://EconPapers.repec.org/RePEc:jae:japmet:v:20:y:2005:i:2:p: $151-159$

Penelope S, Summers PM. 2009. Regime switches in GDP growth and volatility: Some international evidence and implications for modeling business cycles. The B.E. Journal of Macroeconomics 9: 1-19.

Schotman P, van Dijk HK. 1991a. A Bayesian analysis of the unit root in real exchange rates. Journal of Econometrics 49: 195-238.

URL http://ideas.repec.org/a/eee/econom/v49y1991i1-2p195-238.html

Schotman PC, van Dijk HK. 1991b. On Bayesian routes to unit roots. Journal of Applied Econometrics 6: 387-401.

URL http://ideas.repec.org/a/jae/japmet/v6y1991i4p387-401.html 